Civic Reception at the Town Hall on the evening of 23 April, by invitation of the Mayor and Corporation of Scarborough.

The Ulster Cup competition was held at the Ganton

Golf Club on 24 April, and was won by Dr. R. J. Young. The competition for the Cup presented by Dr. B. E.
Schlesinger and Professor C. E. Dent, to be called the U.C.H. Squash Cup, was held at the Scarborough Lawn Tennis and Squash Club on 24 April and was won by Professor C. E. Dent.

The Annual Dinner was held on the evening of 24 April, with Mr. Hugh Greenwood as guest of honour.

\title{
Therapeutic Trials in Wilms' Tumour (Nephroblastoma) and Neuroblastoma
}

The Medical Research Council Working Party on Embryonal Tumours in Childhood (Chairman Professor R. S. Illingworth) is currently planning two therapeutic trials in Wilms' tumour (nephroblastoma) and neuroblastoma.

The trial of treatment in nephroblastoma is designed to assess the relative efficacy of vincristine and actinomycin $\mathrm{D}$ as agents in the treatment of nephroblastoma following surgery and radiotherapy. There is now increasing evidence that the prognosis of nephroblastoma is improved by the use of actinomycin $\mathrm{D}$ for periods of two years following surgery and radiotherapy. There is also evidence that vincristine, a drug which is somewhat less toxic than actinomycin $D$, is efficacious in treating metastatic disease. It is therefore planned to conduct a controlled clinical trial in children over the age of 1 year with Stage I, IIA, and IIB (American classification) disease.

The trial of treatment of neuroblastoma is designed to investigate whether the regression that sometimes occurs in this disease is a consequence of an immune reaction developed against the tumour and if so whether this immunity can be increased by immunotherapy. There is experimental evidence that neuroblastomas contain tumour specific antigens against which an immune reaction may be stimulated, and also that the immune system is more likely to succeed in eradicating a tumour when the number of residual malignant cells in the body is low. It is therefore important that immunotherapy should follow a period of chemotherapy, and in this trial it is proposed to treat patients with vincristine and cyclophosphamide for this purpose. The Working Party considered that the most suitable group of patients for such a trial are as follows.

(1) All patients over the age of 1 year with neuroblastoma, except those with a primary lesion confined to the cervical region.

(2) All children under the age of 1 year with metastatic disease.

Owing to the rarity of these tumours and the importance of obtaining information on the best methods of treatment with reasonable speed, it is hoped that suitable cases will be notified to the secretaries of the Working Party:-Dr. P. Morris Jones and Dr. Dorothy Pearson (at The Royal Manchester Children's Hospital, Pendlebury, Manchester. M27 1HA), from whom further information and detailed protocols may be obtained. 